ISSN: 2527-1075

\title{
AVALIAÇÃO SENSORIAL E VIABILIDADE COMERCIAL DE UM COOKIE ENRIQUECIDO COM $\beta$-GLICANA
}

\section{SENSORY EVALUATION AND COMMERCIAL FEASIBILITY OF A $\beta$-GLYCANE ENRICHED COOKIE}

\author{
T. R. BABOS ${ }^{1}$, M. V. ABRANCHES ${ }^{1}$, R. L. SALES ${ }^{1}$, M. O. BARBOSA ${ }^{1}$ \\ Universidade Federal de Viçosa, Campus Rio Paranaíba. Instituto de Ciências Biológicas e \\ da Saúde \\ E-mail: meire.barbosa@ufv.br
}

\author{
article info \\ Article history: \\ Received 12 May 2017 \\ Accepted 3 August 2017 \\ Available online 20 September 2017
}

PALAVRAS-CHAVE: Análise sensorial; Cookies; $\beta$-glicana; Farelo de aveia.

\begin{abstract}
KEYWORDS: Sensory analysis; Cookies; B-glican; Oat bran.
\end{abstract}
RESUMO: A alimentação é uma necessidade básica e vital para a sobrevivência humana, e o desenvolvimento de produtos mais saudáveis é de grande importância. Nesse contexto, o objetivo desse trabalho foi desenvolver um biscoito tipo cookie rico em $\beta$-glicana proveniente do farelo de aveia e avaliar sua aceitação, bem como sua viabilidade comercial. Foram elaboradas duas receitas de cookies: uma sem adição de $\beta$-glicana (padrão) e outra com adição de $3 \%(p / p)$ de $\beta$-glicana (teste) proveniente da substituição parcial da farinha branca por farelo de aveia. A análise sensorial das preparações foi realizada por 30 provadores não treinados e utilizou-se a escala hedônica de nove pontos para os atributos "Cor", "Textura", "Sabor" e "Impressão global”. Para o teste de intenção de compra foi utilizada uma escala estruturada de cinco pontos, variando de "certamente compraria" a "certamente não compraria". Em relação à composição química, o cookie teste apresentou menor valor calórico (305,6 Kcal/100g), menor percentual de carboidratos (42,6 g/100g), maior percentual de proteínas $(9,8 \mathrm{~g} / 100 \mathrm{~g})$, além de ser fonte de fibras $(5,4 \mathrm{~g} / 100 \mathrm{~g})$, em relação ao cookie padrão. A análise sensorial demonstrou que ambos os cookies foram aceitos, não apresentando diferença estatística $(p<0,05)$. Quanto à intensão de compra a maioria dos provadores compraria o produto se o mesmo estivesse à venda. $O$ produto obtido apresenta características de viabilidade comercial como um produto rico em fibras.

\begin{abstract}
Food is a basic and vital necessity for human survival, and the development of healthier products is of great importance. In this context, the objective of this work was to develop a cookie-type rich in $\beta$-glycans from oat bran and to evaluate its acceptance, as well as its commercial viability. Two cookie recipes were prepared: one without addition of $\beta$-glycan (standard) and another with addition of $3 \%(w / w)$ of $\beta$-glycan (experimental) from the partial replacement of white flour with oat bran. The sensory analysis was performed by 30 untrained tasters and the nine-point hedonic scale was used for the attributes "Color", "Texture", "Flavor" and "Global printing". The experimenatl cookie had a lower caloric value (305.6 Kcal / 100g), a lower percentage of carbohydrates $(42.6 \mathrm{~g} / 100 \mathrm{~g})$, a higher percentage of proteins $(9.8 \mathrm{~g} / 100 \mathrm{~g})$ and a source of fiber $(5.4 \mathrm{~g} / 100 \mathrm{~g})$ when compared to the standard cookie $(P<0.05)$. The purchase intent was positive for the experimental cookie. The product obtained has commercial viability characteristics as a fiber-rich product.
\end{abstract}

\section{INTRODUÇÃ̃}

A alimentação constitui uma necessidade básica e vital para sobrevivência humana, onde $\mathrm{o}$ ato de se alimentar não representa apenas a ingestão de elementos nutritivos, mas 


\section{The Journal of Engineering and Exact Sciences - \\ JCEC \\ ISSN: 2527-1075}

também engloba fatores socioculturais que levam em consideração fatores religiosos, ecológicos, econômicos, psicológicos, afetivos, emocionais (MARTINS, 2011).

Atualmente, a saúde pública mundial vem enfrentando um grande desafio com o aumento das Doenças Crônicas Não Transmissíveis (DCNT). Estudos demonstram que as mudanças no padrão alimentar e no estilo de vida vêm contribuindo para a obesidade, o qual tem sido considerado uma das principais causas para o desenvolvimento das DCNT (AZEVEDO et al., 2014). O objetivo do presente estudo foi desenvolver um biscoito tipo cookie rico em $\beta$-glicana, proveniente do farelo de aveia e de menor aporte energético e avaliar sua aceitação utilizando análise sensorial e sua viabilidade comercial.

\section{MATERIAL E MÉTODOS}

Trata-se de uma pesquisa de caráter experimental, randomizado, transversal. Foram elaboradas duas receitas de cookies sendo uma sem a adição de $\beta$-glicana (cookie padrão) e a outra com a adição de $3 \%(\mathrm{p} / \mathrm{p})$ de $\beta$-glicana (cookie teste) proveniente da substituição parcial da farinha branca por farelo de aveia. Além da adição da fibra, foram realizadas outras substituições, dentre elas o açúcar por adoçante; a água substituindo o leite; e para minimizar as diferenças visuais e melhorar a palatabilidade foi adicionado o chocolate em pó.

Os cookies foram preparados de acordo com a metodologia de FASOLIN, et al. (2007), com a adaptações. A composição química foi calculada pela Tabela de Composição de Alimentos (TACO, 2011). A quantidade de $\beta$-glicana foi estimada segundo WEBER (2002).

A análise sensorial dos cookies foi realizada, conforme descrito por AZEVEDO et al. (2011), com adaptações. As duas formulações foram avaliadas quanto à aceitação e intenção de compra, realizado em dois momentos. O primeiro teste foi realizado sem informação prévia a respeito do produto; e no segundo momento do teste o julgador recebeu informações nutricionais a respeito da composição do produto. $\mathrm{O}$ teste de aceitação foi realizado utilizando escala hedônica de nove pontos variando entre os termos "gostei extremamente" e "desgostei extremamente". Os atributos avaliados foram cor, textura, sabor e impressão global. Para o teste de intenção de compra, foi utilizada escala estruturada de cinco pontos, variando de "certamente compraria" a "certamente não compraria" (SOUZA E MENEZES, 2006).

O teste foi realizado no Ambulatório de Atenção Nutricional da Universidade Federal de Viçosa campus Rio Paranaíba e contou com a participação de 30 voluntários não treinados de ambos os sexos que foram recrutados aleatoriamente (LUCIA; MINIM; CARNEIRO, 2010). A pesquisa foi aprovada por Comitê de Ética da Universidade Federal de Viçosa, parecer número 1.185.386 e os julgadores assinarem o Termo de Consentimento Livre e Esclarecido.

Os dados obtidos foram tabulados em uma planilha do Microsoft Office Excel (versão 2010) e para análise estatística foi utilizado o programa SSP realizando a análise de variância ANOVA e testes de Kruskal-Wallis e Wilcoxon a $p<0,05$. 


\section{RESULTADOS E DISCUSSÃO}

A composição química do cookie teste apresentou menor valor calórico e menor percentual de carboidratos, maior percentual de proteínas, e pode ser considerado como fonte de fibras (Tabela 1).

$\mathrm{Na}$ análise sensorial observou-se que para nenhum dos atributos avaliados houve diferença estatística significativa $(p>0,05)$ entre as duas formulações dos cookies, nos dois momentos. De acordo com as notas obtidas, pôde-se verificar que as duas formulações de cookies (padrão e teste) foram aceitas; com medianas entre 2 e 3 equivalentes a gostei muito e gostei moderadamente, respectivamente, considerando todos os atributos (Tabela 2).

Em relação à influência da informação nutricional na análise sensorial das preparações (padrão e teste), não foi verificada diferença estatística (Tabela 3).

Tabela 1 - Composição química em $100 \mathrm{~g}$ da cookie padrão e cookie teste, Rio Paranaíba MG, 2016

\begin{tabular}{ccccccc}
\hline Cookies & Kcal & Proteína & Lipídio & Carboidrato & $\begin{array}{c}\text { Fibras } \\
\text { totais }\end{array}$ & $\boldsymbol{\beta}$-Glicanas \\
\hline $\begin{array}{c}\text { Cookie } \\
\text { padrão }\end{array}$ & 413,9 & 5,6 & 14,7 & 64,2 & 1,4 & - \\
$\begin{array}{c}\text { Cookie } \\
\text { teste }\end{array}$ & 305,6 & 9,8 & 14,9 & 42,6 & 5,7 & 2,5
\end{tabular}

Fonte: Weber F. H., Gutkoski L. C., Elias M. C. (2002); Taco (2011).

Tabela 2 - Teste de associação entre os cookies padrão com e sem informação nutricional e cookies teste com e sem informação nutricional, Rio Paranaíba, 2016

\begin{tabular}{lllllll}
\hline Atributos & & \multicolumn{5}{c}{ Cookies } \\
& CPSI* & CPCI** & $P$ & CTSI*** & CTCI**** & $P$ \\
& $\begin{array}{l}\text { Mediana } \\
\text { (min-máx.) }\end{array}$ & $\begin{array}{l}\text { Mediana } \\
\text { (min-máx.) }\end{array}$ & & $\begin{array}{l}\text { Mediana } \\
\text { (min-máx.) }\end{array}$ & $\begin{array}{l}\text { Mediana } \\
\text { (min-máx.) }\end{array}$ \\
\hline Cor & $2(1-8)$ & $2(1-8)$ & 0,5 & $2(1-8)$ & $2(1-8)$ & 0,813 \\
\hline Textura & $2(1-9)$ & $3(1-9)$ & 0,063 & $2(1-8)$ & $2,5(1-8)$ & 0,625 \\
\hline Sabor & $2(1-9)$ & $2,5(1-9)$ & 1,0 & $3(1-9)$ & $2(1-9)$ & 0,094 \\
\hline $\begin{array}{l}\text { Impressão } \\
\text { global }\end{array}$ & $2,5(1-9)$ & $3(1-9)$ & 1,0 & $3(1-9)$ & $3(1-9)$ & 0,129 \\
\hline
\end{tabular}

Teste estatístico: Wilcoxon $\mathrm{p}<0,05$.

* CPSI- Cookie Padrão Sem Informação; **CPCI- Cookie Padrão Com Informação

***CTSI- Cookie Teste Sem Informação; ****CTCI- Cookie Teste Com Informação.

O teste de atitude de compra, aos mesmos participantes do teste anterior, mostrou que no primeiro momento (antes da informação nutricional), a maioria dos provadores $(50 \%)$ 


\section{The Journal of Engineering and Exact Sciences - \\ JCEC}

ISSN: 2527-1075

informa que compraria o cookie padrão, enquanto $36,66 \%$ comprariam o cookie teste. Para o cookie padrão não houve alteração na intenção de compra após a informação nutricional enquanto para o cookie teste houve um aumento de $10 \%$ na intenção de compra.

O desenvolvimento de produtos que possam agregar mais de um benefício à alimentação saudável tem sido objeto de vários estudos. A indústria vem investindo cada vez mais na produção de alimentos light (redução no mínimo 25\% de determinados nutrientes gordura saturada, gordura total, açúcar, colesterol, sódio), podendo estes produtos ser destinados às pessoas com algum distúrbio no metabolismo ou consumidores preocupados com a alimentação saudável e com menos calorias (MANHANI et al, 2014). MOTA et al (2011) verificaram que um bolo adicionado de polidextrose e inulina foi bem aceito no teste de aceitação sensorial e com bom potencial de comercialização, podendo ser caracterizada como alimento "light", "diet", com "baixo teor de gorduras" e "alto teor de fibras".

Tabela 3 - Teste de aceitabilidade dos atributos avaliados na análise sensorial dos cookies padrão e teste, antes e após informação nutricional do produto, Rio Paranaíba-MG, 2016

\begin{tabular}{|c|c|c|c|c|c|c|}
\hline \multirow[t]{3}{*}{ Atributos } & \multicolumn{6}{|c|}{ Cookies } \\
\hline & \multicolumn{3}{|c|}{ Sem Informação } & \multicolumn{3}{|c|}{ Com Informação } \\
\hline & $\begin{array}{l}\text { Cookie } \\
\text { padrão } \\
\text { Mediana } \\
\text { (min- } \\
\text { máx.) }\end{array}$ & $\begin{array}{l}\text { Cookie teste } \\
\text { Mediana } \\
\text { (min-máx.) }\end{array}$ & $P$ & $\begin{array}{l}\text { Cookie } \\
\text { padrão } \\
\text { Mediana } \\
\text { (min-máx.) }\end{array}$ & $\begin{array}{l}\text { Cookie } \\
\text { teste } \\
\text { Mediana } \\
\text { (min- } \\
\text { máx.) }\end{array}$ & 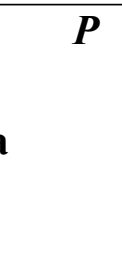 \\
\hline Cor & $2(1-8)$ & $2(1-8)$ & 0,336 & $2(1-8)$ & $2(1-8)$ & 0,200 \\
\hline Textura & $2(1-9)$ & $2(1-8)$ & 0,814 & $3(1-9)$ & $2,5(1-8)$ & 0,418 \\
\hline Sabor & $2(1-9)$ & $3(1-9)$ & 0,833 & $2,5(1-9)$ & $2(1-9)$ & 0,700 \\
\hline $\begin{array}{l}\text { Impressão } \\
\text { global }\end{array}$ & $2,5(1-9)$ & $3(1-9)$ & 0,958 & $3(1-9)$ & $3(1-9)$ & 0,499 \\
\hline
\end{tabular}

Teste estatístico: Kruskal-Wallis. $\mathrm{p}<0,05$.

Segundo a Portaria $n^{\circ} .27$, de 13/01/98, da Secretaria de Vigilância Sanitária do Ministério da Saúde, produtos fonte de fibras ou com teor aumentado em fibras são aqueles produtos que contem na sua formulação o mínimo de $3 \mathrm{~g}$ de fibras em $100 \mathrm{~g}$ de produto (BRASIL, 2001) . A literatura apresenta muitos trabalhos que buscam o desenvolvimento de produtos fonte de fibras, como formulação de biscoitos ricos em fibras por acréscimo de aveia e bagaço de uva (PIOVESANA E BUENO, 2013); massas de pizza com farinha de trigo integral e de linhaça (BOSSONI, 2012).

Os resultados obtidos nesse estudo corroboram com a literatura, uma vez que o cookie teste pode ser classificado como "light" (redução de $27 \%$ de calorias) e "fonte de fibras" $(5,7 \mathrm{~g}$ por $100 \mathrm{~g}$ de produto). Ressalta-se ainda que os atributos avaliados na análise sensorial apresentassem mediana entre gostei muito e gostei moderadamente sem diferenças estatísticas entre os dois produtos (padrão e teste). 


\section{The Journal of Engineering and Exact Sciences - \\ JCEC \\ ISSN: 2527-1075}

GUIMARÃES et al (2010), afirmam que a adição de farinhas de produtos não convencionais com farinha de trigo, podem melhorar suas características nutricionais e possuir uma boa aceitação pelos consumidores. No presente estudo, dados referentes intenção de compra foi positivo. Pôde ainda ser observado que após o recebimento da informação nutricional, o percentual de intenção de compra para cookie teste aumentou (10\%), o que demonstra a preocupação dos provadores com a manutenção e/ou prevenção da saúde.

\section{CONCLUSÕES}

O produto desenvolvido - cookies enriquecidos com fibras (aveia) foi bem aceito e apresenta propriedades sensoriais semelhantes ao cookie padrão, tornando-se alternativa mais saudável na tecnologia de desenvolvimento de novos produtos. No teste de intenção de compra a maioria dos participantes apontou que compraria o cookie padrão tal como o cookie teste, caso estivesse no mercado. A substituição de outros ingredientes no cookie reduziu valor calórico, o que possibilita o acesso a uma população que necessita alimentos especiais. Assim, o produto obtido apresenta características de viabilidade comercial e que ainda poderá auxiliar no controle e prevenção de DCNT.

\section{REFERÊNCIAS}

AZEVEDO, F. L. A. A. et al. Avaliação sensorial de pão de forma elaborado com soro de leite em pó. Revista Brasileira de Produtos Agroindustriais, Campina Grande, v.13, n.1, p.37-47, 2011.

AZEVEDO, E. C. C.; et al. Padrão alimentar de risco para as doenças crônicas não transmissíveis e sua associação com a gordura corporal- uma revisão sistemática. Ciência \& saúde coletiva, v. 19, n. 5, p. 1447-1458, 2014.

BUSSONI, R. C. et. al. Aceitabilidade sensorial de massa de pizza acrescida de farinhas de trigo integral e de linhaça (Linum usitatissimum L.) entre adolescentes. Revista do Instituto Adolfo Lutz (Impresso), v.71, n.3, p. 481-487, 2012.

BRASIL. Ministério da Saúde. Agência Nacional de Vigilância Sanitária. RDC no 40, de 21 de março de 2001. Regulamento técnico para rotulagem nutricional obrigatória de alimentos e bebidas embaladas. Diário Oficial da União.

CARUSO, L.; MENEZES, E. W. Índice glicêmico dos alimentos. Nutrire, v. 19, n. único, p. 49-64, 2000.

FASOLIN, L. H. et al. Biscoitos produzidos com farinha de banana: avaliações química, física e sensorial. Ciência e Tecnologia de Alimentos, v. 27, n. 3, p. 524-529, 2007.

GUIMARÃES, R. R.; FREITAS, M. C. J.; SILVA, V. L. M. . Bolos simples elaborados com farinha da entrecasca de melancia (Citrullus vulgaris, sobral): avaliação química, física e sensorial. Ciência e Tecnologia Alimentar. v. 30, n . 2, p. 354-363, 2010.

LUCIA, S. M. D.; MINIM, V. P. R.; CARNEIRO, J. D. S. Análise sensorial de alimentos. In: MINIM, V. P. R. Análise sensorial: estudos com consumidores. 2. ed. Viçosa, MG: editora. UFV, 2010. Cap. 1, p. 13-49.

MANHANI, T. M.; CAMPOS, M. V. M.; DONATI, F. P. Sacarose, suas propriedades e os novos Edulcorantes. Revista Uniara, v. 17, n. 1, 2014.

MARTINS, M. C. A. A alimentação humana e a Enfermagem: em busca de uma dietética compreensiva. Revista de Enfermagem Referência, v.4, p.143-149, 2011. 
MOTA, A. C.; ClARETO, S. S.; AZEVEDO, E. M. C.; ALMEIDA D. M.; MORAIS A. L. L. Bolo light, diet e com alto teor de fibras: elaboração do produto utilizando polidextrose e inulina. Revista Instituto Adolfo Lutz (Impresso), v.70, n.3, p. 268-275, 2011.

PIOVESANA, A.; BUENO, M. M. Elaboração e aceitabilidade de biscoitos enriquecidos com aveia e farinha de bagaço de uva. Brazilian Journal of Food Technology. Campinas, v.16, n.1, p. 68-72, 2013.

SOUZA, M. L.; MENEZES, H. C. de. Avaliação sensorial de cereais matinais de castanhado-brasil com mandioca extrusados1. Ciência e Tecnologia de Alimentos. Campinas, v. 26, n. 4, p. 950-955, 2006.

TACO. Tabela brasileira de composição de alimentos. Núcleo de Estudos e pesquisas em Alimentação. 4. ed. rev. e ampl. Campinas, 161 p. 2011.

WEBER, F. H.; GUTKOSKI, L. C.; ELIAS, M. C. Caracterização química de cariopses de aveia (Avena sativa L) da cultivar. UPF 18. Ciência e Tecnologia de Alimentos, v.22, n.1, p. 39-44, 2002. 